\title{
A Mechatronic Device for Spasticity Quantification
}

\author{
João Ferreira ${ }^{1}$, Vitor Moreira ${ }^{2}$, José Machado ${ }^{3}$, IEEE Member, Filomena Soares ${ }^{1}$, IEEE Member \\ ${ }^{1}$ University of Minho, Electronics Department, Algoritmi Research Centre, Guimarães, Portugal \\ j.f.ferreira@hotmail.com,fsoares@dei.uminho.pt \\ ${ }^{2}$ Braga Hospital, Braga, Portugal \\ vitorvarandasmoreira@gmail.com
}

${ }^{3}$ University of Minho, Mechanical Engineering Department, СT2M Research Centre, Guimarães, Portugal jmachado@dem.uminho.pt

\begin{abstract}
Spasticity is a common and complex motor disorder that affects more than $\mathbf{1 2}$ million persons in the world. There are several studies on spasticity quantification in the literature but there is still a need for measurement improvements. This paper presents the design of a mechatronic device for spasticity quantification, in joint of ankle, elbow and knees. This approach is based on the velocity dependent of the tonic stretch reflexes. The relevant variables, the measurement range and the adequate measurement systems are selected. The data acquisition system, board and software, are also defined and tested in laboratory. The proposed system was tested in rehabilitation clinical environment and the corresponding results are presented in this article.
\end{abstract}

\section{INTRODUCTION}

Spasticity is a complex motor disorder due to a supra-spinal inhibition, resulting from a hyper-excitability of the stretch reflex (SR) [1]. Spasticity affects more than 12 million persons in the world [2], and is always seen in patient with upper motor neuron dysfunctions such as cerebral vascular accidents, spinal cord injuries, and multiple sclerosis. The mechanism of spasticity is commonly thought as an exaggerated SR, which is a velocity-dependent increase in the resistance to the passive movement [3]. The SR Threshold is significantly reduced in spastic muscles, and this reduction is correlated to the increase in reflex joint torque [4]. Spasticity in conjugation with excessive muscle tone frequently interferes in the voluntary motor function, causing difficulties in daily activities [3]. Some of the common symptoms are: a change in the recruitment of limb's segments and a severe mal-functioning of the tendons reflex.

The correct quantification of spasticity has been under an extensive study by the scientific community, but there is not yet available a well-accepted standard method for spasticity determination and quantification. The literature presents several methods for spasticity quantification: The Ashworth Scale (AS) and the Ashworth modified version (MAS); Isokinetic device with torque generator; Pendulum Test. None of them is fully accepted, due to various reasons [2], [5], [6]. The AS and the MAS are the common scales in clinical quantifications of spasticity, despite experts agree that both scales may not measure the characteristics that distinguish spasticity from other tonus disorders. Although the scale is useful in determining the amount of resistance felt in the passive displacement of the limbs, it does not quantify the dependence to velocity, which is the feature that differentiates spasticity [5]. This scale has a low reproductive rate, a lack of validation in all muscle groups, usually affected by spasticity [1]. The approach of traditional measures is based on the phase and magnitude of the tonic SR and the resistance to passive stretch. Nevertheless, this measure is not correlated to the clinical impression of the spasticity degree, inability to differentiate the mechanical stiffness from the reflective stiffness and the implementation of the device is still complex; also, the measurement does not meet the criteria of the known theory. Hence, there is still a need for a device that meets these requirements [1], [5], [6].

The key issue is to determine which variables are necessary to correctly quantify this disorder. A correct measure to quantify spasticity must follow the physiological mechanisms related to the stand-up position control and the movement in healthy individuals and/or must detect possible deficiencies in any of these mechanisms that lead to motor disorders. For the acceptance of the method, their approach must be in accordance with a standard spasticity definition [5], [6]. The works presented in [7] defined spasticity as: "a motor disorder characterized by a velocity-dependent increase in tonic stretch reflexes ("muscle tonus") with exaggerated tendon jerk, resulting in hyper excitability of the stretch reflexes, as one of the component of the upper motor neuron syndrome". This definition is still accepted nowadays; it includes some important aspects: it refers spasticity as a symptom, as a disorder in the somatic mobility, related to the high tonic component of the SR; it is due to the spinal reflex; it is one of the symptoms of the upper motoneuron syndrome; the tonic stretch is associated to the exaggerated tendon jerks, and reflects the physical component of the SR; the reflex of the tone stretch is the basis of the tonus; it is referred that the excess of the reflex depends on the stretch velocity [8]. This last statement is the key issue for spasticity quantification [2].

This paper presents the study on the design of a mechatronic device for quantification of all levels of spasticity, in joint of ankle, elbow or knees. This approach is based on the velocity dependent increase in the tonic SR, 
according to the criteria of spasticity definition proposed by Lance [7], to establish the relationship, for clinical evaluation, between all levels of spasticity.

Mechatronic devices integrate mechanical and electronic engineering solutions. More specifically, it is a multidisciplinary approach to design new products and manufacturing systems working in a variety of environmentsand in different domain applications. Furthermore, the objective is to design devices that are more efficient, less costly, more friendly and easier to use. Besides the applications in traditional areas as factory, office and home automation, new and very useful applications on rehabilitation domain [9], in finding generic biomedical solutions and/or specific biomedical solutions such as artificial hearts [10], haptic virtual reality environments [11], artificial limbs [12] that interface with the wearer's nervous system, among others.

The article is divided in four sections: section I introduces the spasticity concept and the problem formulation; section II presents the proposed approach used for spasticity quantification; section III discusses the obtained results and explains related ongoing works and, finally, section IV resumes the conclusions.

\section{PROPOSED APPROACH}

The SR is an involuntary contraction elicited by a brief stimulus to muscle receptors. If the arm and muscle are immobilized the result will be a measurable change in the tension of the tendon [4].

The objective of this work is to develop a device for the quantification of all levels of spasticity, which can be accepted by scientific community. It aims to develop a universal device that allows the evaluation for the joint of ankle, knees or elbow. On this approach the method is focused on the velocity dependent increasing in the Tonic SR, according to the criteria of standard definition of Spasticity, proposed by Lance [7]. This definition suggested that the Stretch Reflex Threshold (SRT) depends on the velocity of stretch.

It is determined the angle of biomechanics range and the angular velocity when an increase in electromyography activity occurs, for further data processing, by a custom developed program. Most of the daily life activity requires joint angles of $45^{\circ}$ for ankle, $140^{\circ}$ to full flexion to the knee [13] and $170^{\circ}$ for elbow [14]. These ranges should be considered to ensure the assessment of movement in the whole biomechanical range, recruited in daily life. The study proposed in [2], where are studied patients with stroke, there is detected an increase of activity of the EMG (electromyography) signal in Biceps Brachia, due to stretching the elbow joint, at angular velocities of $51 \%$, $161 \%$ and $430 \%$; this demonstrates the dependence of velocity of SR, in muscle affected by spasticity.

To determine the SR Threshold we propose three measurements: EMG signal activity in the muscle; angular velocity of passive muscle stretch and the joint angle position.
Table 1 resumes the parameters and the measuring ranges for the proposed equipment (Figure 1).

TABLE 1:

VARIABLE MEASURING RANGE IN THE PROPOSED APPROACH

\begin{tabular}{cc}
\hline Parameters & Measuring range \\
\hline angle & $180^{\circ}$ \\
electromyography & $20-500 \mathrm{~Hz}$ \\
angular velocity & $500^{\circ} / \mathrm{s}$
\end{tabular}

\section{A. Experimental setup}

The tests were performed in Fisimaia rehabilitation clinic. Twenty five patients (18 men and 7 women), with diagnosed spasticity symptoms have participated in the study. Table 2 presents the characterization of these patients.

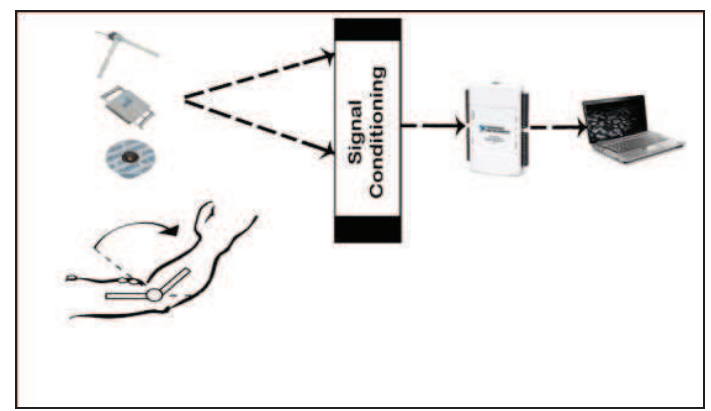

Fig. 1: Proposed system for spasticity quantification.

TABLE 2

CHARACTERIZATION DATA OF THE STUDIED PATIENTS

\begin{tabular}{l|ccccl}
\hline Subject & Age & Gender & Montlinjury & Sidelinjury & \multicolumn{1}{c}{ Injury } \\
\hline M3 & 71 & M & 101 & R & $\begin{array}{l}\text { Post- } \\
\text { stroke }\end{array}$ \\
M6 & 37 & M & 10 & L & $\begin{array}{l}\text { Post- } \\
\text { stroke }\end{array}$ \\
M8 & 76 & M & 8 & L & $\begin{array}{l}\text { Post- } \\
\text { stroke }\end{array}$ \\
M13 & 16 & F & 201 & R & $\begin{array}{l}\text { Cerebral } \\
\text { Palsy }\end{array}$ \\
\hline
\end{tabular}

After being informed about implications risks and benefits of their participation in the study, it was obtained the written Informed Consent of each participant. There have been selected patients with levels of spasticity in the elbow flexor muscles (biceps). It has been assured that patients were not under any medication treatment that could have possible implication in the results. There have been excluded, also, participants who presented pain or cognitive restrictions that limit their ability to collaborate in the study.

The tests were performed by an instructor with indicated capabilities for the task, well trained by a neurologist specialist during the period of approximately one year that preceded the tests, in order to guarantee the success of their elaboration. 
The experimental tests, in each patient, were performed during three different sessions, with an interval from two to four days. During each session, the patients were evaluated three times, giving a global number of 9 times for each patient, if we consider the three sessions. All the patients who showed symptoms of fatigue were evaluated only two times on the next sessions. Also, each patient was evaluated always at the same time - in the morning or in the afternoon - in order to decrease the potential fluctuations in the response of spastic muscles, which could influence the results.

The experimental set-up protocol consists of the following steps:

The patient sits on a chair and the electromyography sensors are fixed on a motor point of a biceps brachial and the EMG signal at rest (tone) is recorded. To record the biomechanical angle, biomechanical range and compute the angular velocity a goneometer is fixed to the arm of the individual. The axis of rotation of the goneometer is placed in correspondence to the elbow joint. The arm of goneometer is aligned and fixed to the arm and forearm of the patients.

The patient is placed in the ideal joint position. The initial angle position and biomechanical range of joint are recorded and constantly monitored, to ensure maximum repeatability and maximal Reflex response of the Biceps Brachial.

In each stretch the initial angle joint is checked and only starts a new stretch if this condition is confirmed. The beginning of each stretch only occurs if the EMG signal, in the initial position, corresponds to the signal captured at rest, (the muscle tone).

\section{B. Experimental procedure}

In order to determining the dynamic SRT (DSRT), the muscle is stretched manually at different velocities. DSRT is defined as the joint angle and the corresponding velocity value, when the EMG signal amplitude also increases with the velocity, above the threshold corresponding with the EMG signal amplitude at rest. The Tonic SRT (TSRT) represents a specific value of DSRT when velocity equals to zero (at rest). In this approach, the DSRT and the TSRT are expressed in velocity and angular coordinates values. The SRT can be expressed as a specific point in the range of joint angle. Thus the SRT can be related to the body frame of reference.

In Figure 2 the DSRT is plotted on a coordinate system, in two dimensions, angular velocity versus angle. Regression analyses can be used in order to compute TSRT value, by extrapolating the regression line through the points of DSRT. When the regression line crosses the axes corresponding at velocity value equal to zero, it corresponds to the coordinate angle at rest (TSRT).

For each linear regression, it was obtained the $r^{2}$ and it was considered a prediction interval of $95 \%$ (Figure 3 ), for the distribution of the results. The DSRT that does not belong to the considered prediction interval are considered "false detections" and were classified as "technical error" that occurred as consequence of artifacts associated to the system or by fluctuations in the movement promoted by the evaluator.

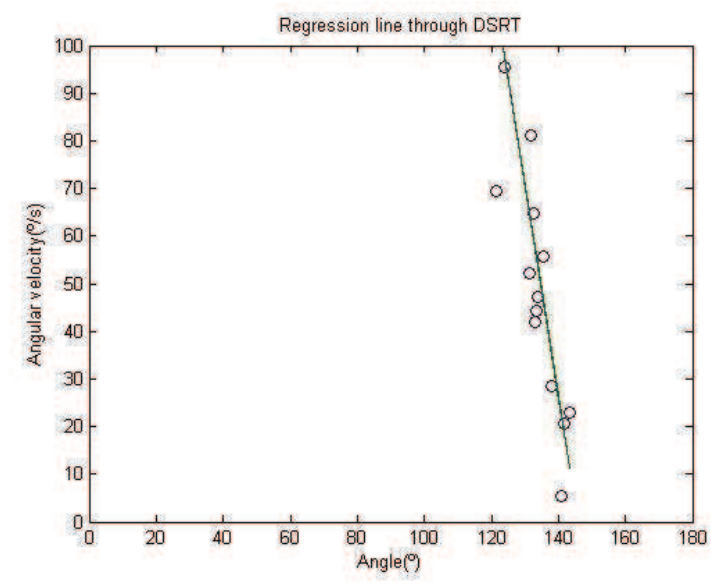

Fig: 2 Example of the TSRT estimation from the patient M6; calculation of the regression line trough the regression line of the DSRT that intersects the $\mathrm{X}$ axis at the value of $145^{\circ}$ and presents a $\mathrm{r}^{2}=0.7075$.

Figure 4 shows the relation between the range of regulation of TSRT and biomechanical range of the joint [1]. The grey areas to right of diagonal lines indicate the areas where spastic muscle is active [2], [17] Previous studies with animals and humans suggest that in healthy individuals the range of regulation $\lambda$ (TSRT) beyond biomechanical range of joint $(\theta-, \theta+)$, where $\theta$ - is the start position of the passive stretch and $\theta+$ is the end position of stretch. In sick individuals the range of regulation $\lambda$ lies within the biomechanical range of joint.

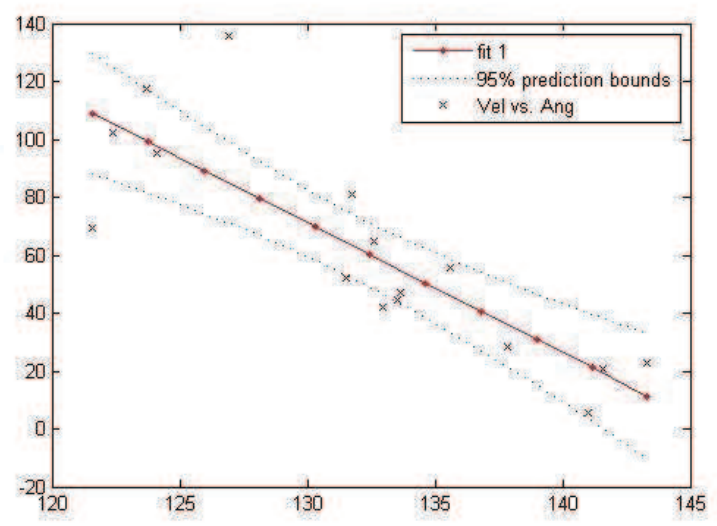

Fig. 3 Data distribution of the DSRT, concerning patient M6, in comparison with linear regression, with 4 DSRT out of the considered range.

\section{EMG Signal detection}

Electromyography records the electrical activity of muscle, and it is a powerful tool in the analysis of human muscular system. When the muscles are active they produce an electric current generally proportional to muscle activity. EMG studies the muscle function through the interpretation of bioelectric signal produced by the muscle. 


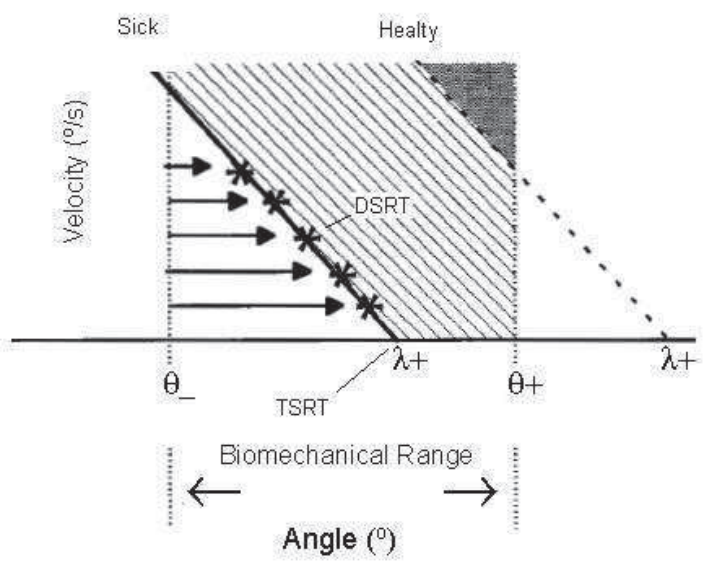

Fig: 4 Relation between range of regulation of STRT and biomechanical range of joint. Adapted from [17].

To measure the EMG signal, surface or needle electrodes are used, depending on the muscle type, superficial or deep. Passive surface electrodes have no amplification in the electrode; active surface electrodes have a signal preamplification system before being sent to the conditioner, which enable a noise reduction.

In this approach, passive surface $\mathrm{Ag} / \mathrm{AgCL}$ electrodes are used and they do not cause pain to the patient. The SENIAM [15] recommends the use of electrodes $\mathrm{Ag} / \mathrm{AgCl}$, together with a conductive gel to reduce signal noise by ensuring a better contact between the electrode and the skin. The signal muscle when measured using surface electrodes has amplitude to $5 \mathrm{mV}$. The frequency range of the EMG signal for the correct analysis is limited between $20 \mathrm{~Hz}$ and $500 \mathrm{~Hz}$, since frequencies below $20 \mathrm{~Hz}$ tend to fluctuate and to be unstable.

The electrodes have a bipolar configuration, enabling a high rate of common mode rejection, and easily eliminating/reducing signal noise. They should be placed in a $20 \mathrm{~mm}$ distance from each other. A surface cleaning gel should also be used in order to reduce the impedance skin/electrode.

The raw EMG signal is detected, amplified and sampled with an analog to digital converter (ADC), after filtering with an anti-aliasing filter. The detection algorithm is implemented in LabVIEW Software, from National Instrument so that the user can monitor the procedure and the results. The EMG signal increase detection is calculated by the method proposed in [16].

Precise detection of on-off timing of human skeletal muscle during movement, based on surface electromyography (sEMG), is an important issue in the analysis of the motor system. The results depend on the chaise of threshold. The goal is to perform signal estimation from noise contaminated EMG signal. The common method for resolving motor-related events from EMG signals consists of visual impression by trained observers. The "single-threshold method", which compares the EMG signal with a fixed threshold, is generally unsatisfactory. Double threshold detection method proposed by [16] is better than single-threshold because it yields higher detection probability and higher sensitivity. Double-detection allows the user to adapt the link between false alarm and detection probability with a higher degree of freedom than the singlethreshold [17], [18]. The onset time EMG detection algorithm is implemented in software Matlab from MathWorks.

\section{RESULTS AND DISCUSSION}

The important data for spasticity quantification are, as already indicated: angular velocity, joint angle, electromyography signal activity and detection of time onset EMG activation in the muscle. A precise data acquisition related to these electrical signals can assure that the proposed mechatronic device is working as expected and, also, that is able to allow quantifying spasticity according to the previous presented methods.

In Figure 5 it can be seen in (a) the sEMG signal recorded in the biceps of a healthy person, which has four muscle contractions, and in (b) the on-off response of the developed program to detect muscle recruitment. In this approach, the logical level is set to 0 when the muscular activity corresponds to the muscular tone (muscle activity at rest) and placed at the logical level 1 when the muscle activity increases in relation to muscular tone. The time instant when the logic level goes from 0 to 1 corresponds to muscle activation.

In this example, the algorithm was successfully able to distinguish the different muscle contractions.

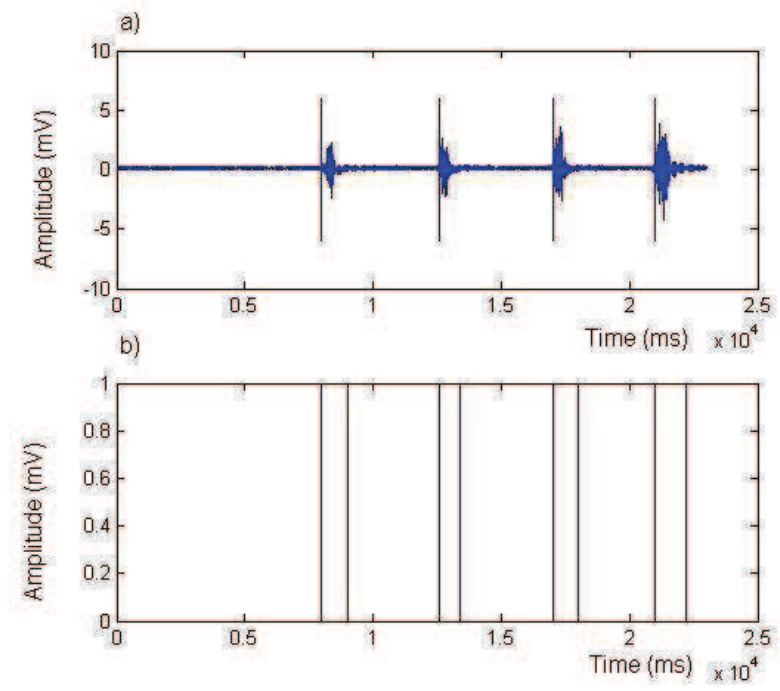

Fig.5. Detection results for biceps (a) original raw EMG signal; (b) results of proposed method on-off detection

Figure 6 shows the graphs corresponding to the angular displacement (a), angular velocity (b) and EMG activity of the muscle studied (c). These graphs were recorded during a test performed on patient M6, when it was promoted a 


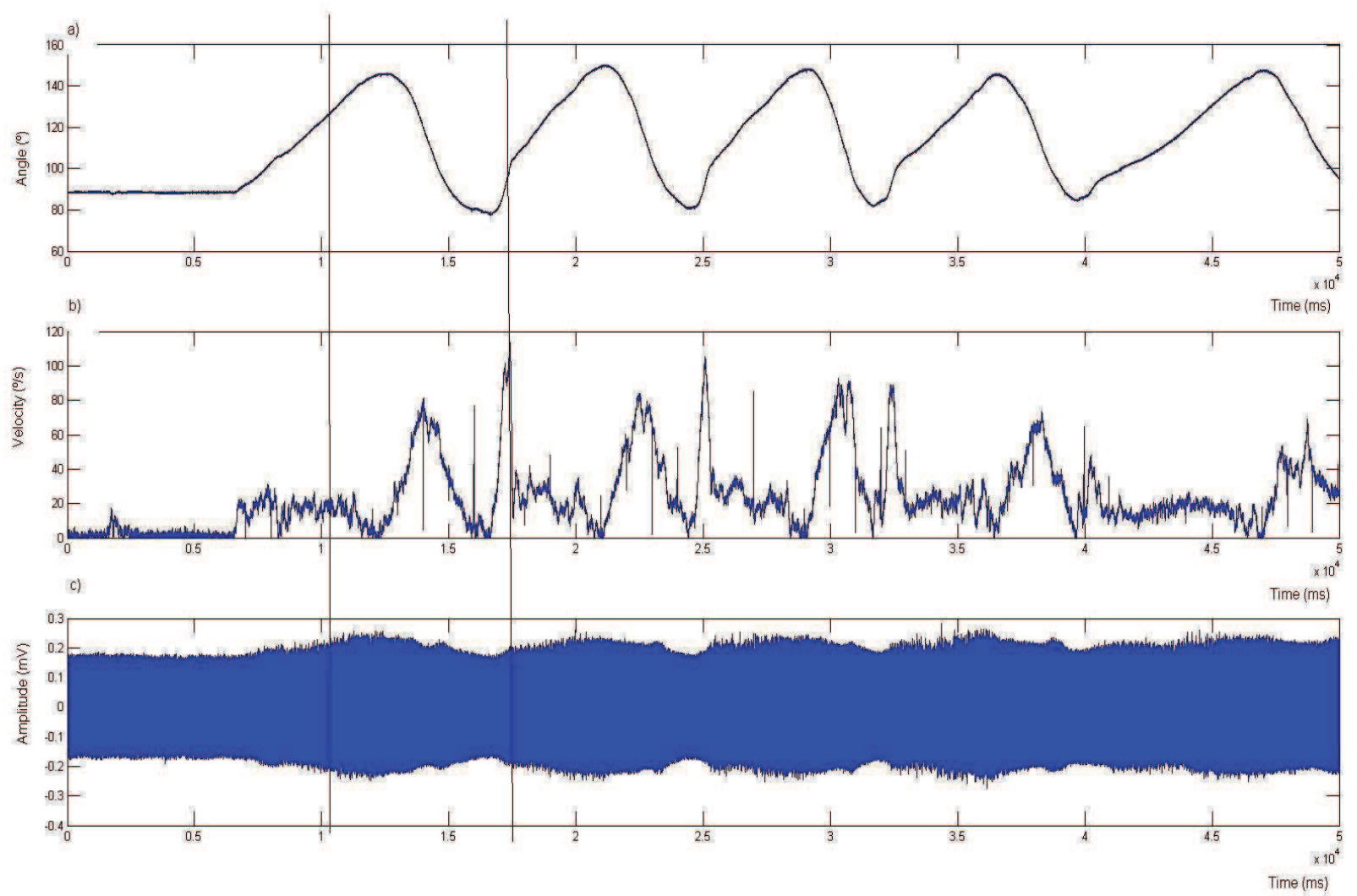

Fig.6. Example of detection of the biceps DSRT patient M6, by passive stretch at different velocities: angular displacement (a), angular velocity (b) and EMG activity of the muscle studied (c).

muscle strain at different velocities of stretch of the elbow flexor muscles.

Vertical lines show the relationship between the time instant where there is an increase of muscle activity, revealing the occurrence of a SR, the angular velocity at which the muscle was being stretched in that time instant and its joint amplitude.

Following the vertical lines in Figure 6, it appears that the low velocities of stretch $\left(19,58^{\circ} / \mathrm{s}\right)$ SR occurs at an amplitude of $127^{\circ}$. While at a higher velocity of stretch $(114.6 \%$ s $) \mathrm{SR}$ occurs at a range of motion of $90^{\circ}$, clearly confirming the dependence on the SR speed. The combination of the angular amplitude values and angular velocity, which occurs in muscle activation, defines the DSRT.

As expected, the SR responded linearly to muscle stretch proportionally to stretch velocity. Figure 2 illustrates an example of TSRT estimation, considering a patient named M6. It can be observed the distribution of 13 DSRTs represented as small circles that allowed the calculation of the regression line, applying the proposed methodology. It can be stated that the regression line intersects the axis corresponding to the angular displacement at the value $145^{\circ}$, clearly inside the range of movements of the joint. In this case, the regression line presents a slope of -13.6 and a correlation coefficient of 0.7075 .

This method allows an objective evaluation of the range of motion affected by spasticity, depending on the speed of muscle stretching, as opposed to subjective methods most often used. In Figure 2, it can be observed that the patient has only a range of motion up to $145^{\circ}$ from the range of joint movements. The area to the left of the regression line expresses the range of motion in terms of speed that the patient has. While the area right of the line expresses the range of motion in which the patient shows no movement.

The DSRT have been identified in all the patients and the evaluations that presented a DSRT value less than 6 were rejected. Also, the evaluation' results in which the DSRT dispersion and the regression line present $r^{2}<0.1$ were rejected. Table 3 presents the obtained results in two evaluations with patients M3, M6, M8 and M13.

TABLE 3

RESULTS OBTAINED IN TWO SESSIONS

\begin{tabular}{l|cccccc}
\hline Subject & $\frac{\text { Test 1 }}{\text { TSRT }}$ & $r^{2}$ & Slope & $\frac{\text { Test 2 }}{\text { TSRT }}$ & $r^{2}$ & Slope \\
& 193 & 0.78 & -2.73 & 203 & 0.1 & -2.856 \\
M3 & 149 & 0.6033 & -2.189 & 145 & 0.7075 & -4.488 \\
M6 & 154 & 0.6092 & -4.56 & 156 & 0.6704 & -8.872 \\
M13 & 166 & 0.3812 & -6.557 & 156 & 0.4684 & -5.9408 \\
\hline
\end{tabular}


The patients M6 and M8 show $\mathrm{r}^{2}$ close to 1 , in the first and also in the second test. These results show reliability of the calculations on the regression line in function of the DSRT. Although the values of $r^{2}$ of the patient M13 are lower, they present satisfactory values. It can be stated that, concerning the patients M6, M8 and M13, $\mathrm{r}^{2}$ reveals low fluctuation, when comparing the tests. The values of TSRT also present low fluctuation between the first and the second tests.

\section{CONCLUSIONS AND FUTURE WORK}

Literature presents some studies related to spasticity quantification and development of dedicated devices for this goal. All these studies highlight that spasticity quantification is a key subject and it is very important to develop reliable systems to accomplish correct spasticity quantification.

The spasticity quantification based on the dependence of the velocity of the stretch reflex threshold reflects the characteristics of the spasticity, according to the recognized definitions for this problem. There are no doubts that this method is the most adequate because it considers all the spasticity characteristics. Even so, a study based on the evaluation of the variation of velocity can present a low fluctuation between results from different evaluations.

This work shows satisfactory results using this method and, also, shows that the developed mechatronic device for spasticity quantification was a successful task and it gives the desired and expected results, with repeatability.

Although this, with this system, it is now, possible to calculate, with precision the correct doses of toxin to administrate at each patient, without mistakes.

\section{ACKNOWLEDGMENTS}

The authors are grateful to Portuguese Research Centers Algoritmi and CT2M for financial support. The authors are also grateful to Fisimaia rehabilitation clinic in Maia and his patients.

\section{REFERENCES}

[1] P. Le Carvozin, X. Hemot, O. Bartier, G. Carrault, F. Chagneau, P. Callien, H. Allain, P. Rochcongar, Evaluation of pendulum testing of spasticity, in Annales de la Réadaptation et de Médecine Physiqu, Mars. 2002, 45, pp. 510-516.
[2] A. Calota, A.G. Feldman, M.F. Levin, Spasticity measurement based on tonic stretch reflex threshold in stroke using a portable device. In Clinical Neurophysiology. 2008, 119, pp. 2329-2337.

[3] J.J Chen, Y.N. Wu, S.C Huang, H.M. Lee and Y.L. Wangs, The Use of a Portable Muscle Tone Measurement Device to Measure the Effects of Botulinum Toxin Type A on Elbow Flexor Spasticity. Arch Phys Med Rehabil,. Vol. 86, August. 2005.

[4] M. Chardon, Nina L, Surech and w.z. Ryner. A New Method For Reflex Threshold Estimation in Spastic Muscles. Presented at the conference of the IEEE EMBS. Sep. 2009.

[5] M.F. Levin, On the nature and measurement of spasticity, in Clinical Neurophysiology, 2005. 116; 1754-1755

[6] J L. Bernhard, Towards a Clinical measurement of Spasticity. In Physiotherapy. 199581.

[7] J.W. Lance, Spasticity: Disorder of Motor Control. Chicago, Year Book Medical, 1980, pp. 485-500.

[8] P. Decq, P. Pilipetti, J.P. Lefaucheur, Evaluation of Spasticity in Adults. Operative Technique in Neurosurgery. 2005, PP. 100-108.

[9] G. Bucca, A. Bezzolato, S. Bruni, F. Molteni. A Mechatronic Device for the Rehabilitation of Ankle Motor Function. J. Biomech. Eng. December 2009 -- Volume 131, Issue 12, 125001 (7 pages), doi:10.1115/1.4000083.

[10] C.M. Cavalheiro, D. Fo, A. Andrade, J. Cardoso, O. Horikawa, E. Bock. Artificial Organs Volume 35, Issue 5 (2011-05-01), pp. 465470.

[11] K. Vlachos, A haptic tele-manipulation environment for a vibrationdriven micromechatronic device. 2007 IEEE/ASME International conference on Advanced Intelligent Mechatronics, Vols. 1-3 : 1100$110,52007$.

[12] P. Aravinthan, N. GopalaKrishnan, P.A. Srinivas, N. Vigneswaran. Emerging Trends in Robotics and Communication Technologies (INTERACT), 2010 International Conference on 2010 pages: 241 244

[13] L. Febiger, Kinesiology and Applied Anatomy (7th ed.), Guanabara Koogans. A. 1989, PP. 146-170.

[14] O. Ennis, D. C. Miller, P. Kelly,. Fracture of the adult elbow, in Current Orthopaedics. 2008, 22; pp. 111-131.

[15] H. J. Hermens, B.Freriks, C. Disselhorst-Klug, G. Rau, Development of recommendations for SEMG sensors and sensor placement procedures. In Journal of Electromyography and Kinesiology. 2000, $10 ;$ pp. 361-374.

[16] X. Lanyi, A. Adler, An improved method for muscle activation detection during gait. Canadian Conference of Electrical and Computer Engineering 2004..

[17] A.Jobin and M.E.Levin. Regulation of stretch reflex threshold in elbow flexors in children with cerebral palsy: a new measure of spasticity. Developmental Medicine \& Child Neurology. 2000, 42; pp. 531-540.

[18] M.B.I. Reaz, M.S.S Hussain and F.Mohd-yasin. Techniques of EMG signal analysis: detection, processing, classification and aplication.in Biol. Proced. Online. 2006; 8(1):11-35. 